Tom 25 № 2

\title{
КОНСТАНТЫ НИКОЛЬСКОГО - БЕРНШТЕЙНА ДЛЯ ЦЕЛЫХ ФУНКЦИЙ ЭКСПОНЕНЦИАЛЬНОГО СФЕРИЧЕСКОГО ТИПА В ВЕСОВЫХ ПРОСТРАНСТВАХ ${ }^{1}$
}

\author{
Д. В. Горбачев, В. И. Иванов
}

\begin{abstract}
Мы изучаем точную константу в неравенстве Никольского - Бернштейна $\|D f\|_{q} \leq C\|f\|_{p}$ на подпространстве целых функций $f$ экспоненциального сферического типа в пространстве $L^{p}\left(\mathbb{R}^{d}\right)$ с весом $v_{\kappa}$ степенного типа. В качестве дифференциального оператора $D$ рассматривается неотрицательная целая степень лапласиана Данкля $\Delta_{\kappa}$, ассоциированного с весом $v_{\kappa}$. Этим также охватывается одномерный случай пространства $L^{p}\left(\mathbb{R}_{+}\right)$со степенным весом $t^{2 \alpha+1}$ и дифференциальным оператором Бесселя. Наш основной результат заключается в доказательстве равенства между многомерной и одномерной весовыми константами при $1 \leq p \leq q=\infty$. Для этого мы показываем, что норма $\|D f\|_{\infty}$ может быть заменена значением $D f(0)$, что было известно только в одномерном случае. Необходимое отображение подпространства функций, по сути сводящее задачу к радиальной, а значит, одномерной, осуществляется при помощи положительного оператора обобщенного сдвига Данкля $T_{\kappa}^{t}$. Мы доказываем его новое свойство аналитического продолжения по переменной $t$. Как следствие мы вычисляем весовую константу Бернштейна при $p=q=\infty$, которая была известна в исключительных случаях. Также мы приводим некоторые оценки констант и даем небольшой список открытых проблем.
\end{abstract}

Ключевые слова: неравенство Никольского - Бернштейна, точная константа, целая функция экспоненциального сферического типа, вес степенного типа, лапласиан Данкля.

D. V. Gorbachev, V. I. Ivanov. Nikol'skii-Bernstein constants for entire functions of exponential spherical type in weighted spaces.

We study the exact constant in the Nikol'skii-Bernstein inequality $\|D f\|_{q} \leq C\|f\|_{p}$ on the subspace of entire functions $f$ of exponential spherical type in the space $L^{p}\left(\mathbb{R}^{d}\right)$ with a power-type weight $v_{\kappa}$. For the differential operator $D$, we take a nonnegative integer power of the Dunkl Laplacian $\Delta_{\kappa}$ associated with the weight $v_{\kappa}$. This situation encompasses the one-dimensional case of the space $L^{p}\left(\mathbb{R}_{+}\right)$with the power weight $t^{2 \alpha+1}$ and Bessel differential operator. Our main result consists in the proof of an equality between the multidimensional and one-dimensional weight constants for $1 \leq p \leq q=\infty$. For this, we show that the norm $\|D f\|_{\infty}$ can be replaced by the value $D f(0)$, which was known only in the one-dimensional case. The required mapping of the subspace of functions, which actually reduces the problem to the radial and, hence, one-dimensional case, is implemented by means of the positive operator of generalized Dunkl translation $T_{\kappa}^{t}$. We prove its new property of analytic continuation in the variable $t$. As a consequence, we calculate the weighted Bernstein constant for $p=q=\infty$, which was known in exceptional cases only. We also find some estimates of the constant and give a short list of open problems.

Keywords: Nikol'skii-Bernstein inequality, exact constant, entire function of exponential spherical type, power-type weight, Dunkl Laplacian.

MSC: 41A17, 42B10

DOI: $10.21538 / 0134-4889-2019-25-2-75-87$

\section{1. Введение}

Пусть $d \in \mathbb{N}, \mathbb{R}^{d}-d$-мерное евклидово пространство со скалярным произведением $\langle x, y\rangle=$ $x_{1} y_{1}+\ldots+x_{d} y_{d}$ и нормой векторов $|x|=\sqrt{\langle x, x\rangle}, B^{d}=\left\{x \in \mathbb{R}^{d}:|x| \leq 1\right\}$ - единичный шар. Для $0<p \leq \infty$ и положительной на $Q \subset \mathbb{R}^{d}$ меры $d \rho$ через $L^{p}(Q, d \rho)$ будем обозначать (весовое) пространство измеримых функций $f: Q \rightarrow \mathbb{C}$ с конечной нормой $\|f\|_{p, d \rho}=$ $\left(\int_{\mathbb{R}^{d}}|f(x)|^{p} d \rho(x)\right)^{1 / p}$ при $p<\infty$ (квази-нормой при $\left.p<1\right)$ или $\|f\|_{\infty}=\operatorname{ess}_{\sup } \in Q|f(x)|$ при $p=\infty$. Как обычно, $d x$ для лебеговой меры опускается и $L^{\infty}(Q)=C(Q)$ для непрерывных

\footnotetext{
${ }^{1}$ Исследование выполнено за счет гранта Российского научного фонда (проект 18-11-00199).
} 
функций. Через $C$ будем обозначать положительные константы, которые могут меняться от места к месту. Пусть $\lceil a\rceil$ - наименьшее целое, не меньшее $a$.

В классических неравенствах Бернштейна, Никольского, Маркова для подпространств полиномов и целых функций экспоненциального типа $L^{q}$-норма функции или ее производной оценивается через $L^{p}$-норму и порядок или тип функций соответственно (см. обзоры результатов в $[3 ; 5 ; 6])$. Данные неравенства играют важную роль во многих вопросах гармонического анализа и его приложений, особенно в теории приближений. Большой интерес представляют как порядковые неравенства с точным порядком роста, так и неравенства с точными константами. Последний случай интересен также тем, что конструкция экстремальной функции часто учитывает геометрические характеристики пространства, на котором заданы функции. Иногда этот эффект позволяет решить сложную геометрическую задачу.

Точная константа Никольского - Бернштейна в неравенстве $\|D f\|_{q, d \rho} \leq C\|f\|_{p, d \rho}$ для подпространства $Y \subset L^{p}(Q, d \rho)$ и дифференциального оператора $D$ определяется равенством

$$
\mathcal{K}_{p, q}(Y, D)=\sup _{f \in Y \backslash\{0\}} \frac{\|D f\|_{q, d \rho}}{\|f\|_{p, d \rho}}, \quad 0<p, q \leq \infty .
$$

Если $D=I$ - тождественный оператор, то константу принято называть константой Никольского (иногда Джексона - Никольского), а если $D \neq I, p=q$, то - константой Бернштейна.

Мы изучаем задачу о точной константе Никольского-Бернштейна для подпространств целых функций экспоненциального сферического типа в пространствах $L^{p}\left(\mathbb{R}^{d}, v_{\kappa}(x) d x\right)$ с весом $v_{\kappa}$ степенного типа и лапласианом Данкля $\Delta_{\kappa}$, что охватывает безвесовой случай.

Мы рассмотрим случай $p \leq q=\infty$ и докажем, что норму $\|D f\|_{\infty}$ можно заменить значением в нуле $D f(0)$. Этот факт активно изучался, например, в работах авторов [3]. Важность возможности сдвига точки максимума в нуль обусловлена несколькими причинами. Например, 1) с его помощью доказывается предельная связь между тригонометрической константой Никольского-Бернштейна и константой для целых функций экспоненциального типа при $p<q=\infty$, полученная в работах [6;15 $(d=1) ; 5(d \geq 1)]$. В [15, разд. 3] этот факт специально обсуждается в контексте гипотезы о предельной связи при $q<\infty$. 2) В нашем случае сдвиг позволит свести многомерную задачу к одномерной весовой задаче и установить факт существования экстремальной функции. 3) Становится возможным использовать общие оценки в одномерной задаче для многомерной константы. В частности, таким образом мы вычислим весовую константу Бернштейна при $p=q=\infty$.

1. Приведем один из многомерных результатов о связи констант, вытекающий из $[5$, теорема 1.3]. Пусть $\mathcal{T}_{n}^{d}-$ множество тригонометрических полиномов $t(x)=\sum_{k \in \mathbb{Z}^{d} \cap n B^{d}} c_{k} e^{i\langle k, x\rangle}$ порядка $n>0, \mathcal{E}_{\sigma}^{d}$ - множество целых функций экспоненциального сферического типа не больше $\sigma>0, \Delta-$ оператор Лапласа и для $r \in \mathbb{Z}_{+}$

$$
\mathcal{C}_{d}(n, p, q, r)=\mathcal{K}_{p, q}\left(\mathcal{T}_{n}^{d} \cap L^{p}\left((-\pi, \pi]^{d}\right), \Delta^{r}\right), \quad \mathcal{L}_{d}(p, q, r)=\mathcal{K}_{p, q}\left(\mathcal{E}_{1}^{d} \cap L^{p}\left(\mathbb{R}^{d}\right), \Delta^{r}\right)
$$

- соответствующие константы Никольского - Бернштейна. Тогда при $d \in \mathbb{N}, 0<p \leq q=\infty$

$$
\lim _{n \rightarrow \infty} \frac{\mathcal{C}_{d}(n, p, \infty, r)}{n^{2 r+d / p}}=\mathcal{L}_{d}(p, \infty, r)
$$

Случай $q<\infty$ сложнее, и в нем установлена только оценка сверху (гипотетически правильная). В работе [5] доказан общий случай спектра полиномов и функций, задаваемого при помощи центрально симметричного выпуклого тела с центром в нуле, и однородного дифференциального оператора с постоянными коэффициентами. Интересно отметить, что равенство, аналогичное (1.1), справедливо и для полиномов на евклидовой сфере $\mathbb{S}^{d}[4]$.

При $d=1$ равенство (1.1) было ранее получено в работах [15 $(r=0) ; 6(r \geq 0)]$. Поведение остаточного члена при $r=0$ можно оценить, например, из границ [8]

$$
n^{1 / p} \leq \frac{\mathcal{C}_{1}(n, p, \infty, 0)}{\mathcal{L}_{1}(p, \infty, 0)} \leq\left(n+\left\lceil p^{-1}\right\rceil\right)^{1 / p}, \quad n \in \mathbb{Z}_{+}, \quad 0<p<\infty .
$$


Обзор точных констант Никольского - Бернштейна для подпространств тригонометрических полиномов и функций экспоненциального типа см., например, в [6 $(d=1) ; 5(d>1)]$. В общем случае точные константы известны при $(p, q) \in\{(2,2),(2, \infty)\}$, когда достаточно воспользоваться неравенством Коши - Буняковского для производной воспроизводящего ядра подпространства. Для $d=1$ и $0<p=q \leq \infty$ имеем классическую константу Бернштейна, равную 1. Для $d>1$ константа Бернштейна

$$
\mathcal{L}_{d}(\infty, \infty, 1)=d
$$

вычислена в работе [13]. В статьях [11;13] и др. эти результаты обобщены на случай компактных однородных пространств. Отметим предельный случай $p=0$, где одномерные тригонометрические константы Никольского - Бернштейна получены в [1;2]. Во всех остальных ситуациях задача представляется открытой.

Наилучшая известная нам равномерная верхняя граница константы Никольского для целых функций экспоненциального сферического типа имеет вид [16]

$$
\mathcal{L}_{d}(p, q, 0) \leq\left(\left(\frac{\lceil p / 2\rceil}{2 \pi}\right)^{d}\left|B^{d}\right|\right)^{1 / p-1 / q}, \quad 0<p \leq q<\infty, \quad d \in \mathbb{N},
$$

где $\left|B^{d}\right|=\pi^{d / 2} / \Gamma(d / 2+1)$ - объем шара $B^{d}$. Эта оценка точная только при $(p, q)=(2, \infty)$. Однако она дает отправную точку для исследования асимптотического поведения константы Никольского $\mathcal{L}_{d}(p, q, 0)$, в частности, при больших размерностях $d$. Кроме того, в [13] наряду c (1.2) показано, что

$$
\mathcal{L}_{d}(p, p, 1) \leq d, \quad 1 \leq p \leq \infty .
$$

Однако, как отмечает автор, эти оценки при $1 \leq p<\infty$ требуют уточнения, так как, например, при $p=2$ и $d>1$ имеем $\mathcal{L}_{d}(2,2,1)=1$.

2. Как известно, множество целых функций экспоненциального сферического типа обладает сферической симметрией, т. е. инвариантно относительно действия группы вращений $O(d)$. Поэтому представляет интерес рассмотреть задачу о точной константе Никольского - Бернштейна на подмножестве радиальных функций $f(x)=f_{0}(|x|)$, которые инвариантны относительно этого действия. Более того, при $q=\infty$ и $1 \leq p \leq \infty$ общая и радиальная задачи оказываются эквивалентными. Мы не можем дать прямую ссылку на этот факт, однако далее он будет доказан даже в большей общности для весовых пространств $L^{p}\left(\mathbb{R}^{d}\right)$ (см. теорему 1$)$.

Как известно, подмножество радиальных функций в $L^{p}\left(\mathbb{R}^{d}\right)$ изометрично множеству четных функций в $L^{p}\left(\mathbb{R}_{+}, t^{d / 2-1} d t\right)$. Поэтому константа Никольского - Бернштейна для радиальных функций сводится к соответствующей задаче для четных целых функций экспоненциального типа на полуоси с весом $t^{2 \alpha+1}, \alpha \geq-1 / 2$. Пусть для $t \in \mathbb{R}_{+}$

$$
d \nu_{\alpha}(t)=b_{\alpha} t^{2 \alpha+1} d t, \quad b_{\alpha}^{-1}=2^{\alpha} \Gamma(\alpha+1), \quad D_{\alpha}=\frac{d^{2}}{d t^{2}}+\frac{2 \alpha+1}{t} \frac{d}{d t},
$$

где $D_{\alpha}$ - дифференциальный оператор Бесселя, который при $\alpha=d / 2-1$ совпадает с радиальной частью оператора Лапласа.

Пусть $\mathcal{B}_{p, d \nu_{\alpha}}^{\sigma}\left(\mathbb{R}_{+}\right)$- подпространство $L^{p}\left(\mathbb{R}_{+}, d \nu_{\alpha}\right)$ четных целых функций экспоненциального типа не больше $\sigma>0$. В этом случае точное неравенство Никольского-Бернштейна

$$
\left\|D_{\alpha}^{r} f\right\|_{q, \nu_{\alpha}} \leq \mathcal{L}(\alpha, p, q, r) \sigma^{2 r+(2 \alpha+2)(1 / p-1 / q)}\|f\|_{p, \nu_{\alpha}},
$$

где

$$
\mathcal{L}(\alpha, p, q, r)=\mathcal{K}_{p, q}\left(\mathcal{B}_{p, d \nu_{\alpha}}^{1}\left(\mathbb{R}_{+}\right), D_{\alpha}^{r}\right)
$$

изучалось в работах $[3 ; 18]$.

Настоящая работа является продолжением наших исследований $[4 ; 7-10]$ по по точным и порядковым константам Никольского - Бернштейна в весовых пространствах $L^{p}\left(\mathbb{R}^{d}, v_{\kappa}(x) d x\right)$; 
см. также работу первого автора (Тр. Ин-та математики и механики УрО РАН. 2018. Т. 24, № 4. С. 92-103). В последней работе было доказано, что при $1 \leq p \leq q=\infty$ и $r \geq 0$ неравенство (1.5) с той же константой справедливо для произвольных (необязательно четных) функций из $\mathcal{E}_{\sigma}^{1} \cap$ $L^{p}\left(\mathbb{R},|t|^{2 \alpha+1} d x\right)$ (см. также [7], где изучен случай $r=0$ ), и при $(p, q)=(1, \infty)$ получены некоторые точные результаты на подмножестве неотрицательных функций.

Решение проблемы со сдвигом точки максимума в нуль для константы НикольскогоБернштейна $\mathcal{L}(\alpha, p, \infty, r)$ для $1 \leq p \leq q=\infty$ при $r=0$ вытекает из [3, теорема 2], а при $r \geq 1$ - из теоремы 2 вышеуказанной работы.

Предложение 1. Пусть $\alpha \geq-1 / 2,1 \leq p \leq \infty u r \in \mathbb{Z}_{+}$. Тогда

$$
\mathcal{L}(\alpha, p, \infty, r)=\sup \frac{D_{\alpha}^{r} F(0)}{\|F\|_{p, d \nu_{\alpha}}},
$$

где верхнял грань берется по всем действительным функииям $F \in \mathcal{B}_{p, d \nu_{\alpha}}^{1}\left(\mathbb{R}_{+}\right) \backslash\{0\}$.

Кроме того, существует действительная экстремальная функиия $F_{*} \in \mathcal{B}_{p, d \nu_{\alpha}}^{1}\left(\mathbb{R}_{+}\right)$, такая что $\left\|F_{*}\right\|_{p, d \nu_{\alpha}}=1$ и $D_{\alpha}^{r} F_{*}(0)=\mathcal{L}(\alpha, p, \infty, r)$. При $1<p<\infty$ она единственная.

З а м е ч а н и е 1. В связи с предложением 1 отметим полезное равенство (см. (3.1))

$$
D_{\alpha}^{r} F(0)=\frac{4^{r}\left(\begin{array}{c}
r+\alpha \\
r
\end{array}\right)}{\left(\begin{array}{c}
2 r \\
r
\end{array}\right)} F^{(2 r)}(0) \text {. }
$$

Естественным многомерным обобщением веса $|t|^{2 \alpha+1}$ является вес $\prod_{j=1}^{d}\left|x_{j}\right|^{2 \alpha_{j}+1}$. Однако удобнее рассмотреть более общий случай веса Данкля степенного типа, включающий данный пример. Пусть задан конечный набор векторов $R \subset \mathbb{R}^{d} \backslash\{0\}$, образующих систему корней; $G(R) \subset O(d)$ - конечная группа отражений, порожденная отражениями $\sigma_{a}, a \in R$, относительно гиперплоскостей $\langle a, x\rangle=0 ; \kappa(a): R \rightarrow \mathbb{R}_{+}$- неотрицательная функция кратности, инвариантная относительно группы $G(R)$. Тогда вес Данкля, ассоциированный с $R$, имеет вид

$$
v_{\kappa}(x)=\prod_{a \in R_{+}}|\langle a, x\rangle|^{2 \kappa(a)}, \quad x \in \mathbb{R}^{d},
$$

где $R_{+} \subset R-$ некоторая положительная подсистема системы корней (см. [19]). Например, если $R=\left\{ \pm e_{1}, \ldots, \pm e_{d}\right\}$, где $e_{1}, \ldots, e_{d}$ - единичные орты, то $G(R)=\mathbb{Z}_{2}^{d}$, и мы приходим к отмеченному выше весу $v_{\kappa}(x)=\prod_{j=1}^{d}\left|x_{j}\right|^{2 \kappa_{j}}$, где $\kappa_{j}=\alpha_{j}+1 / 2 \geq 0$.

Положим

$$
d \mu_{\kappa}(x)=c_{\kappa} v_{\kappa}(x) d x, \quad c_{\kappa}^{-1}=\int_{\mathbb{R}^{d}} e^{-|x|^{2} / 2} v_{\kappa}(x) d x,
$$

где нормировочная константа $c_{\kappa}^{-1}$ известна как интеграл Макдональда-Мета-Сельберга. Если функция кратности $\kappa=0$, то получаем безвесовой случай $d \mu_{0}(x)=(2 \pi)^{-d / 2} d x$.

В пространстве $L^{p}\left(\mathbb{R}^{d}, d \mu_{\kappa}\right)$ справедливо свойство однородности

$$
\left\|f_{\lambda}\right\|_{p, d \mu_{\kappa}}=\lambda^{-d_{\kappa} / p}\|f\|_{p, d \mu_{\kappa}}, \quad \lambda>0
$$

где $f_{\lambda}(x)=f(\lambda x)$ и $d_{\kappa}$ - размерность Данкля, определяемая равенством

$$
d_{\kappa}=d+2 \sum_{a \in R_{+}} \kappa(a) .
$$

Роль частных производных играют следующие дифференциально-разностные $G$-инвариантные операторы Данкля:

$$
T_{j} f(x)=\frac{\partial f(x)}{\partial x_{j}}+\sum_{a \in R_{+}} \kappa(a)\left\langle a, e_{j}\right\rangle \frac{f(x)-f\left(\sigma_{a} x\right)}{\langle a, x\rangle}, \quad j=1, \ldots, d .
$$


С их помощью определяются градиент $\nabla_{\kappa}=\left(T_{1}, \ldots, T_{d}\right)$ и лапласиан $\Delta_{\kappa}=\nabla_{\kappa}^{2}$ Данкля. При $\kappa=0$ приходим к обычному градиенту $\nabla$ и оператору Лапласа $\Delta$.

Необходимо отметить, что гармонический анализ в пространстве $L^{p}\left(\mathbb{R}^{d}, d \mu_{\kappa}\right)$ базируется на преобразовании Данкля $\mathcal{F}_{\kappa}$ (см. разд. 2). Для радиальных функций $f(x)=f_{0}(|x|)$ оно сводится к преобразованию Ганкеля $\mathcal{H}_{\alpha_{\kappa}}$ в пространстве $L^{p}\left(\mathbb{R}_{+}, d \nu_{\alpha_{\kappa}}\right)$, где

$$
\alpha_{\kappa}=\frac{d_{\kappa}}{2}-1
$$

При этом

$$
\int_{\mathbb{R}^{d}} f(x) d \mu_{\kappa}(x)=\int_{0}^{\infty} f_{0}(t) d \nu_{\alpha_{\kappa}}(t), \quad \Delta_{\kappa} f(x)=D_{\alpha_{\kappa}} f_{0}(t) .
$$

Пусть $0<p \leq \infty, \sigma>0$. Рассмотрим весовое подпространство

$$
\mathcal{B}_{p, d \mu_{\kappa}}^{\sigma}\left(\mathbb{R}^{d}\right)=\mathcal{E}_{\sigma}^{d} \cap L^{p}\left(\mathbb{R}^{d}, d \mu_{\kappa}\right)
$$

целых функций экспоненциального сферического типа не выше $\sigma$. Напомним, что функции $f$ сферического типа $\sigma$ удовлетворяют неравенству [17, гл. 3]

$$
|f(z)| \leq C_{\varepsilon} e^{(\sigma+\varepsilon)|z|} \quad \forall z \in \mathbb{C}^{d}, \quad \forall \varepsilon>0,
$$

где нижняя грань $\sigma(f)$ называется (точным) сферическим типом $f$. В [9] доказано, что для функций $f \in \mathcal{B}_{p, d \mu_{\kappa}}^{\sigma}\left(\mathbb{R}^{d}\right)$ справедлива более точная оценка

$$
|f(z)| \leq C e^{\sigma|\operatorname{Im} z|} \quad \forall z \in \mathbb{C}^{d} .
$$

Отсюда, в частности, следует, что $f$ ограничена на $\mathbb{R}^{d}$ и $\mathcal{B}_{p, d \mu_{\kappa}}^{\sigma}\left(\mathbb{R}^{d}\right) \subset \mathcal{B}_{\infty}^{\sigma}\left(\mathbb{R}^{d}\right)$ при $0<p<\infty$.

3 а м е ч а н и е 2. С учетом (1.8) для радиальной функции $f \in \mathcal{B}_{p, d \mu_{\kappa}}^{\sigma}\left(\mathbb{R}^{d}\right)$ имеем $f_{0} \in$ $\mathcal{B}_{p, d \nu_{\alpha_{\kappa}}}^{\sigma}\left(\mathbb{R}_{+}\right)$, и наоборот.

В [9, разд. 7] доказано следующее порядковое неравенство Никольского - Бернштейна для лапласиана Данкля:

$$
\left\|\Delta_{\kappa}^{r} f\right\|_{q, d \mu_{\kappa}} \leq C \sigma^{2 r+d_{\kappa}(1 / p-1 / q)}\|f\|_{p, d \mu_{\kappa}}, \quad r \in \mathbb{Z}_{+}, \quad f \in \mathcal{B}_{p, d \mu_{\kappa}}^{\sigma}\left(\mathbb{R}^{d}\right),
$$

где $1 \leq p \leq q \leq \infty$ и $C$ не зависит от $f$ и $\sigma$. Для $d=1, \kappa=\alpha+1 / 2$, четных функций и оператора Бесселя $D_{\alpha}$ получаем неравенство в подпространстве $\mathcal{B}_{p, d \nu_{\alpha}}^{\sigma}\left(\mathbb{R}_{+}\right)$, установленное в [18]. При $p<1$ неравенство (1.9) известно только для $d=1, r \geq 0$ и $d \geq 1, r=0$. Отметим работу [10], где неравенство (1.9) обобщено на случай нецелого $r>0$.

Из соотношений однородности (1.7), $\Delta_{\kappa} f_{\lambda}=\lambda^{2} \Delta_{\kappa} f$ и $\sigma\left(f_{\lambda}\right)=\lambda \sigma(f)$ вытекает, что точную весовую константу Никольского - Бернштейна в неравенстве (1.9) достаточно определить для функций типа не больше 1. Положим

$$
\mathcal{L}_{d, \kappa}(p, q, r)=\mathcal{K}_{p, q}\left(\mathcal{B}_{p, d \mu_{\kappa}}^{1}\left(\mathbb{R}^{d}\right), \Delta_{\kappa}^{r}\right) .
$$

Тогда, например, $\mathcal{L}_{d, 0}(p, q, r)=(2 \pi)^{(d / 2)(1 / p-1 / q)} \mathcal{L}_{d}(p, q, r)$.

Основной результат работы. Сформулируем следующее утверждение о равенстве многомерной (1.10) и одномерной (1.6) констант Никольского - Бернштейна.

Теорема 1. Пусть $d \in \mathbb{N}, \kappa-$ произвольная функиия кратности, $d_{\kappa}=d+2 \sum_{a \in R_{+}} \kappa(a)$, $1 \leq p \leq \infty u r \in \mathbb{Z}_{+}$. Тогда

$$
\mathcal{L}_{d, \kappa}(p, \infty, r)=\mathcal{L}\left(\alpha_{\kappa}, p, \infty, r\right), \quad \alpha_{\kappa}=\frac{d_{\kappa}}{2}-1 .
$$

При этом существует действительная радиальная экстремальная функиия $f_{*}$ такал, что $\left\|f_{*}\right\|_{p, d \mu_{\kappa}}=1 u \Delta_{\kappa}^{r} f_{*}(0)=\mathcal{L}_{d, \kappa}(p, \infty, r)$. 
3 а м е ч а н и е 3 . Для $d=1, \kappa=\alpha+1 / 2$ эта теорема доказана в работе первого автора (Тр. Ин-та математики и механики УрО РАН. 2018). В силу предложения 1 и (1.8), если верно (1.11), то $f_{*}(x)=F_{*}(|x|)$ будет искомой радиальной экстремальной функцией.

3 а м е ч а н и е 4. Доказательство теоремы (1) несложно в безвесовом случае, когда можно воспользоваться инвариантностью подпространства целых функций экспоненциального типа, нормы $\|f\|_{p}$ и оператора Лапласа $\Delta$ относительно оператора сдвига $f(\cdot) \mapsto f\left(\cdot+x_{0}\right)$.

В случае весовых $L^{p}$-пространств, в частности, $L^{p}\left(\mathbb{R}^{d}, d \mu_{\kappa}\right), \kappa \neq 0$, при таком подходе возникает сложность, связанная с тем, что обычный оператор сдвига не является инвариантным для весовой нормы и соответствующего дифференциального оператора. В серии работ авторов [3], посвященных точному неравенству Никольского, для преодоления этой сложности было предложено использовать оператор обобщенного сдвига $T^{t} f(x)$, который действует инвариантно на подпространстве функций $f$ и обладает свойствами $T^{0} f=f$ и $\left\|T^{t} f\right\|_{p} \leq\|f\|_{p}$ при $1 \leq p \leq \infty$. В [9] для общего веса Данкля нами был построен и исследован положительный оператор Данкля $T_{\kappa}^{t}$ (см. разд. 2), который обладает нужными свойствами. Мы установим новый факт действия $T_{\kappa}^{t}$ на подпространстве $\mathcal{B}_{p, d \mu_{\kappa}}^{1}\left(\mathbb{R}^{d}\right)$, который позволит доказать теорему 1 (см. разд. 3).

3. Как следствие теоремы 1 вычислим константу Бернштейна при $p=q=\infty$.

Теорема 2. Для всех $r \in \mathbb{Z}_{+}$

$$
\mathcal{L}_{d, \kappa}(\infty, \infty, r)=\frac{4^{r}\left(\begin{array}{c}
r+\alpha_{\kappa} \\
r
\end{array}\right)}{\left(\begin{array}{c}
2 r \\
r
\end{array}\right)} .
$$

Экстремальной функиией является $(-1)^{r} \cos |x|$.

3 а м е ч а н и е 4 . Имеем $\mathcal{L}_{d, 0}(\infty, \infty, 1)=d$, поэтому эта теорема обобщает равенство $(1.2)$, которое, получается, для вывода точной константы при $r>1$ нельзя итерировать. При $r=1$ теорему 2 можно сразу вывести из $(1.11)$ и равенства $\mathcal{L}(\alpha, \infty, \infty, 1)=2 \alpha+2$ [18].

Далее в разд. 2 мы дадим необходимые сведения из гармонического анализа Данкля и Ганкеля, приведем свойства положительного оператора обобщенного сдвига Данкля и докажем предложение 2 о его аналитическом продолжении. В разд. 3 доказываются теоремы 1 и 2. В заключительном разделе мы приведем некоторые известные нам равенства и оценки для весовых констант Никольского - Бернштейна и предложим несколько открытых задач, часть из которых кажутся нам трудными.

\section{2. Положительный оператор обобщенного сдвига Данкля}

Предварительно нам потребуются некоторые сведения из гармонического анализа Данкля в весовом пространстве $L^{p}\left(\mathbb{R}^{d}, d \mu_{\kappa}\right)$ (см. [9] и ссылки там). Преобразование Данкля определяется равенством

$$
\mathcal{F}_{\kappa}(f)(y)=\int_{\mathbb{R}^{d}} f(x) \overline{e_{\kappa}(x, y)} d \mu_{\kappa}(x), \quad y \in \mathbb{R}^{d},
$$

где $e_{\kappa}(x, y)=E_{\kappa}(x, i y)$ - обобщенная экспонента, $E_{\kappa}(x, y)$ - ядро Данкля.

При $\kappa=0$ имеем $e_{0}(x, y)=e^{i\langle x, y\rangle}$ и обычное преобразование Фурье $\mathcal{F}_{0}$. Другим примером является одномерный случай группы отражений $G=\mathbb{Z}_{2}, \kappa=\alpha+1 / 2$. Тогда получаем пространство $L^{p}\left(\mathbb{R}, 2^{-1} d \nu_{\alpha}\right)$ и $e_{\kappa}(x, y)=e_{\alpha}(x y)$, где мера $d \nu_{\alpha}$ определена в $(1.4)$ и

$$
e_{\alpha}(t)=j_{\alpha}(t)-i j_{\alpha}^{\prime}(t)=j_{\alpha}(t)+\frac{i t}{2(\alpha+1)} j_{\alpha+1}(t)
$$


Здесь $j_{\alpha}(t)=\Gamma(\alpha+1)(2 / t)^{\alpha} J_{\alpha}(t)-$ нормированная функция Бесселя порядка $\alpha, j_{\alpha}(0)=1$. Для нее при $r \in \mathbb{Z}_{+}$имеем

$$
\left(-D_{\alpha, t}\right)^{r} j_{\alpha}(\lambda t)=\lambda^{2 r} j_{\alpha}(\lambda t)
$$

где $D_{\alpha}$ - дифференциальный оператор Бесселя (1.4) и $D_{\alpha, t}$ означает действие по переменной $t$.

Отметим интегральное представление Рёслер $e_{\kappa}(x, y)=\int_{\mathbb{R}^{d}} e^{i\langle\xi, y\rangle} d \mu_{x}^{\kappa}(\xi)$, где $\mu_{x}^{\kappa}-$ вероятностная мера Бореля, носитель которой содержится в выпуклой оболочке $G$-орбиты точки $x$. Это представление дает базовые свойства $e_{\kappa}(x, y)$, аналогичные свойствам $e^{i\langle x, y\rangle}$. Кроме того, обобщенная экспонента является единственным решением дифференциально-разностной задачи $\nabla_{\kappa, x} e_{\kappa}(x, y)=i y e_{\kappa}(x, y), e_{\kappa}(0, y)=1$. Отсюда для лапласиана Данкля

$$
\left(-\Delta_{\kappa, x}\right)^{r} e_{\kappa}(x, y)=|y|^{2 r} e_{\kappa}(x, y) .
$$

Преобразование Данкля $\mathcal{F}_{\kappa}$ является унитарным оператором в пространстве $L^{2}\left(\mathbb{R}^{d}, d \mu_{\kappa}\right)$ и $\mathcal{F}_{\kappa}^{-1}(f)(x)=\mathcal{F}_{\kappa}(f)(-x)$. Оператор $\mathcal{F}_{\kappa}$ автоморфен на классе шварцовских функций $\mathcal{S}\left(\mathbb{R}^{d}\right)$.

Для радиальной функции $f(x)=f_{0}(|x|)$ имеем $\mathcal{F}_{\kappa}(f)(y)=\mathcal{H}_{\alpha_{\kappa}}\left(f_{0}\right)(|y|)$, где $\mathcal{H}_{\alpha}$ - преобразование Ганкеля, определяемое равенством

$$
\mathcal{H}_{\alpha}\left(f_{0}\right)(s)=\int_{\mathbb{R}_{+}} f_{0}(t) j_{\alpha}(s t) d \nu_{\alpha}(t), \quad s \in \mathbb{R}_{+} .
$$

В силу (2.1) преобразование Ганкеля $\mathcal{H}_{\alpha}$ в $L^{p}\left(\mathbb{R}_{+}, d \nu_{\alpha}\right)$ и одномерное преобразование Данкля $\mathcal{F}_{\alpha+1 / 2}$ на подпространстве четных функций из $L^{p}\left(\mathbb{R}, 2^{-1} d \nu_{\alpha}\right)$ совпадают, а значит, обладают аналогичными свойствами. Отсюда и из унитарности $\mathcal{F}_{\alpha+1 / 2}$ в $L^{2}\left(\mathbb{R}, 2^{-1} d \nu_{\alpha}\right)$, например, следует, что оператор $\mathcal{H}_{\alpha}$ унитарный в $L^{2}\left(\mathbb{R}_{+}, d \nu_{\alpha}\right)$ и $\mathcal{H}_{\alpha}^{-1}=\mathcal{H}_{\alpha}$.

Определим положительный оператор обобщенного сдвига (или среднего значения) Данкля $T_{\kappa}^{t}, t \in \mathbb{R}$, и перечислим некоторые его свойства (см. [9]). Пусть $B_{r}(x)$ - шар радиуса $r>0$ с центром в точке $x \in \mathbb{R}^{d}$.

1. Если $f \in C\left(\mathbb{R}^{d}\right)$, то $T_{\kappa}^{t}$ задается формулой Рёслер [19]

$$
T_{\kappa}^{t} f(x)=\int_{\mathbb{R}^{d}} f(z) d \sigma_{x, t}^{\kappa}(z) \in C\left(\mathbb{R}_{+} \times \mathbb{R}^{d}\right),
$$

где $\sigma_{x, t}^{\kappa}-$ вероятностная борелевская мера, для которой

$$
\operatorname{supp} \sigma_{x, t}^{\kappa} \subset \bigcup_{g \in G(R)} B_{t}(g x) \subset B_{|x|+t}(0) .
$$

При этом $T_{\kappa, x}^{t} e_{\kappa}(x, y)=j_{\alpha_{\kappa}}(t|y|) e_{\kappa}(x, y)$. Имеем $\left\|T_{\kappa}^{t}\right\|_{\infty \rightarrow \infty}=1$.

2. В $L^{2}\left(\mathbb{R}^{d}, d \mu_{\kappa}\right)$ оператор $T_{\kappa}^{t}$ определяется как мультипликатор Данкля

$$
T_{\kappa}^{t} f(x)=\int_{\mathbb{R}^{d}} j_{\alpha_{\kappa}}(t|y|) \mathcal{F}_{\kappa}(f)(y) e_{\kappa}(x, y) d \mu_{\kappa}(y) .
$$

Из неравенства $\left|j_{\alpha}(t)\right| \leq j_{\alpha}(0)=1$ следует, что $\left\|T_{\kappa}^{t}\right\|_{2 \rightarrow 2}=1$ для любого $t \in \mathbb{R}_{+}$.

3. Если $f \in \mathcal{S}\left(\mathbb{R}^{d}\right), 1 \leq p<\infty$, то $\left\|T_{\kappa}^{t} f\right\|_{p, d \mu_{\kappa}} \leq\|f\|_{p, d \mu_{\kappa}}$, и оператор $T_{\kappa}^{t}$ продолжается на все пространство $L^{p}\left(\mathbb{R}^{d}, d \mu_{\kappa}\right)$ с сохранением нормы.

4. Оператор $T_{\kappa}^{t}$ четный по $t$, положительный, самосопряженный,

$$
T_{\kappa}^{0} f=f, \quad T_{\kappa}^{t} 1=1, \quad\left\|T_{\kappa}^{t}|f|\right\|_{1, d \mu_{\kappa}}=\|f\|_{1, d \mu_{\kappa}},
$$

откуда $\left\|T_{\kappa}^{t}\right\|_{1 \rightarrow 1}=1$. 
5. Сужение $T_{\kappa}^{t}$ на радиальные функции выражается через оператор обобщенного сдвига Бесселя-Гегенбауэра. При $\kappa=0$ оператор $T_{0}^{t}$ является обычным оператором сферического среднего значения.

6. Пусть $x_{0} \in \mathbb{R}^{d}-$ фиксированная точка. Тогда

$$
\left\|T_{\kappa}^{t} f\left(x_{0}\right)\right\|_{p, d \nu_{\alpha_{\kappa}}}=\left(\int_{\mathbb{R}_{+}}\left|T_{\kappa}^{t} f\left(x_{0}\right)\right|^{p} d \nu_{\alpha_{\kappa}}(t)\right)^{1 / p} \leq\|f\|_{p, d \mu_{\kappa}}, \quad 1 \leq p \leq \infty .
$$

7. В пространстве $C^{\infty}\left(\mathbb{R}^{d}\right)$ оператор $T_{\kappa}^{t}$ перестановочен с $\Delta_{\kappa}$ в том плане, что $D_{\alpha_{\kappa}, t} T_{\kappa, x}^{t}=$ $T_{\kappa, x}^{t} \Delta_{\kappa, x}[14$, предложение 5.2]. Отсюда

$$
D_{\alpha_{\kappa}, t}^{r} T_{\kappa, x}^{t}=T_{\kappa, x}^{t} \Delta_{\kappa, x}^{r}, \quad r \in \mathbb{Z}_{+} .
$$

До сих пор оператор $T_{\kappa}^{t}$ рассматривался для $t \in \mathbb{R}$. Установим следующий результат об аналитическом продолжении $T_{\kappa}^{t}$ на $t \in \mathbb{C}$ для произвольной функции кратности $\kappa$. Тогда

Предложение 2. Пусть $1 \leq p \leq \infty, \sigma>0, x_{0} \in \mathbb{R}^{d}, f \in \mathcal{B}_{p, d \mu_{\kappa}}^{\sigma}\left(\mathbb{R}^{d}\right)$ u $F(t)=T_{\kappa}^{t} f\left(x_{0}\right)$.

$$
F(t) \in \mathcal{B}_{p, d \nu_{\alpha_{\kappa}}}^{\sigma}\left(\mathbb{R}_{+}\right) .
$$

Д о к а з а т е л ь с т в о. В силу (2.7) имеем $\|F\|_{p, d \nu_{\alpha_{\kappa}}} \leq\|f\|_{p, d \mu_{\kappa}}, 1 \leq p \leq \infty$, поэтому достаточно установить, что $F \in \mathcal{B}_{\infty}^{\sigma}\left(\mathbb{R}_{+}\right)$.

Рассмотрим вначале случай подпространства шварцовских функций $f \in \mathcal{B}_{p, d \mu_{\kappa}}^{\sigma}\left(\mathbb{R}^{d}\right) \cap \mathcal{S}\left(\mathbb{R}^{d}\right)$. Тогда по теореме Пэли-Винера [12, теорема 4.10] имеем

$$
f(x)=\int_{B_{\sigma}(0)} \mathcal{F}_{\kappa}(f)(y) e_{\kappa}(x, y) d \mu_{\kappa}(y) .
$$

Отсюда и из $(2.5)$

$$
F(t)=\int_{B_{\sigma}(0)} j_{\alpha_{\kappa}}(t|y|) \mathcal{F}_{\kappa}(f)(y) e_{\kappa}\left(x_{0}, y\right) d \mu_{\kappa}(y) .
$$

Так как $t \mapsto j_{\alpha_{\kappa}}(t|y|)$ - четная целая функция экспоненциального типа $|y| \leq \sigma$, то $F \in \mathcal{B}_{\infty}^{\sigma}\left(\mathbb{R}_{+}\right)$.

Пусть теперь $f \in \mathcal{B}_{p, d \mu_{\kappa}}^{\sigma}\left(\mathbb{R}^{d}\right) \backslash \mathcal{S}\left(\mathbb{R}^{d}\right)$. Построим последовательность шварцовских функций $f_{\varepsilon} \in \mathcal{B}_{\infty}^{\sigma}\left(\mathbb{R}^{d}\right)$, сходящихся равномерно к $f$ при $\varepsilon \rightarrow 0$ на каждом компакте из $\mathbb{R}^{d}$. Для этого при $\varepsilon \in(0,1 / 2)$ можно положить $f_{\varepsilon}(x)=f((1-\varepsilon) x) \psi(\varepsilon x)$, где $\psi \in \mathcal{B}_{\infty}^{\sigma}\left(\mathbb{R}^{d}\right)$ - неотрицательная шварцовская функция, для которой $\|\psi\|_{\infty}=\psi(0)=1$.

Последовательность функций $f_{\varepsilon}$ равномерно ограничена в $L^{p}\left(\mathbb{R}^{d}, d \mu_{\kappa}\right) \cap L^{\infty}\left(\mathbb{R}^{d}\right)$, поскольку с учетом (1.7)

$$
\left\|f_{\varepsilon}\right\|_{p, d \mu_{\kappa}} \leq\|\psi(\varepsilon x)\|_{\infty}\|f((1-\varepsilon) x)\|_{p, d \mu_{\kappa}} \leq(1-\varepsilon)^{-d_{\kappa} / p}\|f\|_{p, d \mu_{\kappa}} \leq 2^{d_{\kappa} / p}\|f\|_{p, d \mu_{\kappa}}
$$

(при $p=\infty$ константа равна 1) и по неравенству Никольского (см. (1.9) при $d=1, r=0$ )

$$
\left\|f_{\varepsilon}\right\|_{\infty} \leq C\left\|f_{\varepsilon}\right\|_{p, d \mu_{\kappa}} .
$$

Положим $F_{\varepsilon}(t)=T_{\kappa}^{t} f_{\varepsilon}\left(x_{0}\right)$ и покажем, что $F_{\varepsilon}(t)$ сходится равномерно к $F(t)$ при $\varepsilon \rightarrow 0$ на каждом компакте $[0, R], R>0$. Действительно, из свойства 1 оператора $T_{\kappa}^{t}$ (см. (2.3) и (2.4)) следует, что

$$
\left|F(t)-F_{\varepsilon}(t)\right| \leq \int_{\mathbb{R}^{d}}\left|f(z)-f_{\varepsilon}(z)\right| d \sigma_{x_{0}, t}^{\kappa}(z) \leq\left\|f-f_{\varepsilon}\right\|_{C\left(B_{\left|x_{0}\right|+t}(0)\right)},
$$


откуда

$$
\left\|F-F_{\varepsilon}\right\|_{C([0, R])} \leq\left\|f-f_{\varepsilon}\right\|_{C\left(B_{\left|x_{0}\right|+R}(0)\right)} \rightarrow 0, \quad \varepsilon \rightarrow 0 .
$$

Для шварцовских функций $f_{\varepsilon}$ мы показали, что $F_{\varepsilon} \in \mathcal{B}_{\infty}^{\sigma}\left(\mathbb{R}_{+}\right)$. При этом, последовательно применяя $(2.7),(2.10)$ и $(2.9)$, получаем

$$
\left\|F_{\varepsilon}\right\|_{\infty} \leq\left\|f_{\varepsilon}\right\|_{\infty} \leq C\left\|f_{\varepsilon}\right\|_{p, d \mu_{\kappa}} \leq C\|f\|_{p, d \mu_{\kappa}},
$$

т. е. последовательность $F_{\varepsilon}$ четных целых функций экспоненциального типа не выше $\sigma$ равномерно ограничена в $L^{\infty}\left(\mathbb{R}_{+}\right)$. Отсюда по теореме компактности для целых функций экспоненциального типа (см., например, $[17,3.3 .6])$ заключаем, что существует подпоследовательность $\left\{F_{\varepsilon_{j}}\right\}_{j=1}^{\infty}$, равномерно сходящаяся при $j \rightarrow \infty$ на каждом отрезке из $\mathbb{R}_{+}$к некоторой функции $F_{*} \in \mathcal{B}_{\infty}^{\sigma}\left(\mathbb{R}_{+}\right)$. Отсюда и из равномерной сходимости на отрезках $F_{\varepsilon}$ к $F$ получаем, что $F$ и $F_{*}$ можно отождествить. Таким образом, $F \in \mathcal{B}_{\infty}^{\sigma}\left(\mathbb{R}_{+}\right)$. Предложение доказано.

\section{3. Доказательство основных результатов}

\section{1. Доказательство теоремы 1}

Докажем равенство (1.11), т. е.

$$
\begin{gathered}
\mathcal{L}_{d, \kappa}(p, \infty, r)=\sup \left\{\frac{\left\|\Delta_{\kappa}^{r} f\right\|_{\infty}}{\|f\|_{p, d \mu_{\kappa}}}: f \in \mathcal{B}_{p, d \mu_{\kappa}}^{1}\left(\mathbb{R}^{d}\right) \backslash\{0\}\right\} \\
=\mathcal{L}(\alpha, p, \infty, r)=\sup \left\{\frac{D_{\alpha}^{r} F(0)}{\|F\|_{p, d \nu_{\alpha}}}: F \in \mathcal{B}_{p, d \nu_{\alpha}}^{1}\left(\mathbb{R}_{+}\right) \backslash\{0\}\right\} .
\end{gathered}
$$

В силу (1.8) и замечания 2 оценка снизу получается на радиальных функциях $f(x)=$ $F(|x|)$. Установим оценку сверху.

Для произвольно малого $\varepsilon>0$ выберем функцию $f \in \mathcal{B}_{p, d \mu_{\kappa}}^{1}\left(\mathbb{R}^{d}\right)$, такую что $\|f\|_{p, d \mu_{\kappa}}=1$ и

$$
\mathcal{L}_{d, \kappa}(p, \infty, r) \leq \Delta_{\kappa}^{r} f\left(x_{0}\right)+\varepsilon
$$

для некоторой точки $x_{0} \in \mathbb{R}^{d}$, зависящей от $\varepsilon$.

Теперь воспользуемся предложением 2 и положим $F(t)=T_{\kappa}^{t} f\left(x_{0}\right)$. Тогда $F(t) \in \mathcal{B}_{p, d \nu_{\alpha_{\kappa}}}^{\sigma}\left(\mathbb{R}_{+}\right)$. В силу (2.7)

$$
\|F\|_{p, d \nu_{\alpha_{\kappa}}} \leq\|f\|_{p, d \mu_{\kappa}}=1
$$

а из $(2.8),(2.6)$

$$
D_{\alpha_{\kappa}, t}^{r} F(t)=T_{\kappa, x}^{t}\left(\Delta_{\kappa, x}^{r} f\right)\left(x_{0}\right), \quad D_{\alpha_{\kappa}, t}^{r} F(0)=\Delta_{\kappa, x}^{r} f\left(x_{0}\right) .
$$

Таким образом, $\mathcal{L}_{d, \kappa}(p, \infty, r) \leq D_{\alpha_{\kappa}, t}^{r} F(0)+\varepsilon$. Функция $F$ зависит от $\varepsilon$, но ясно, что $D_{\alpha_{\kappa}, t}^{r} F(0) \leq \mathcal{L}(\alpha, p, \infty, r)$, поэтому

$$
\mathcal{L}_{d, \kappa}(p, \infty, r) \leq \mathcal{L}(\alpha, p, \infty, r)+\varepsilon
$$

Остается $\varepsilon$ устремить к нулю.

Равенство (1.11) установлено. Существование радиальной экстремальной функции вытекает из замечания 3. Теорема доказана. 


\section{2. Доказательство теоремы 2}

В силу теоремы 1 достаточно показать, что при $\alpha \geq-1 / 2$ и $r \in \mathbb{Z}_{+}$

$$
\mathcal{L}(\alpha, \infty, \infty, r)=\frac{4^{r}\left(\begin{array}{c}
r+\alpha \\
r
\end{array}\right)}{\left(\begin{array}{c}
2 r \\
r
\end{array}\right)}
$$

Пусть $F$ - целая четная действительная целая функция экспоненциального типа не больше 1 , ограниченная на $\mathbb{R}_{+}$. Вычислим $D_{\alpha}^{r} F(0)$ на основе ряда Тейлора

$$
F(t)=\sum_{k=0}^{\infty} \frac{F^{(2 k)}(0)}{(2 k) !} t^{2 k}
$$

В силу (1.4) имеем $D_{\alpha} t^{2 k}=4 k(k+\alpha) t^{2 k-2}$. Отсюда по индукции

$$
D_{\alpha}^{r} t^{2 k}=4^{r} k(k-1) \ldots(k-r+1)(k+\alpha)(k+\alpha-1) \ldots(k+\alpha-r+1) t^{2 k-2 r} .
$$

Таким образом,

$$
D_{\alpha}^{r} F(0)=\frac{4^{r} r ! \Gamma(r+\alpha+1)}{(2 r) ! \Gamma(\alpha+1)}=\frac{4^{r}\left(\begin{array}{c}
r+\alpha \\
r
\end{array}\right)}{\left(\begin{array}{c}
2 r \\
r
\end{array}\right)} F^{(2 r)}(0) .
$$

По классическому неравенству Бернштейна имеем $\left\|F^{(2 r)}\right\|_{\infty} \leq\|F\|_{\infty}$, которое является точным, например, для $\cos t$. Осталось учесть, что $\cos ^{(2 r)}(0)=(-1)^{r}$. Теорема доказана.

\section{4. Финальные замечания}

1. Пусть $\alpha \geq-1 / 2, b_{\alpha}^{-1}=2^{\alpha} \Gamma(\alpha+1)$ - множитель в $d \nu_{\alpha}(1.4), r \in \mathbb{Z}_{+}$. Справедливы следующие результаты для одномерной весовой константы:

1) $\mathcal{L}(\alpha, 2,2, r)=1, \mathcal{L}(\alpha, 2, \infty, r)=\left(\frac{b_{\alpha}}{4 r+2 \alpha+2}\right)^{1 / 2}$ (см. Тр. Ин-та математики и механики УpO PAH. 2018. T. 24, № 4. C. 92-103);

2) $\mathcal{L}(\alpha, p, p, r) \leq 2 \alpha+2, \quad 1 \leq p \leq \infty \quad[18]$;

3) $\mathcal{L}(\alpha, p, \infty, 0) \leq\left(\frac{b_{\alpha}}{2 \alpha+2}\right)^{1 / p}\left\lceil\frac{p}{2}\right\rceil^{(2 \alpha+2) / p}, \quad 0<p<\infty \quad[7]$;

4) $\mathcal{L}(\alpha, p, \infty, 0) \geq\left(\frac{b_{\alpha}}{2 \alpha+2}\right)^{1 / p}\left(\frac{p}{2}\right)^{((2 \alpha+2) / p)(1+o(1))}, \quad \alpha \rightarrow \infty, \quad 0<p<\infty \quad[7]$;

5) $\mathcal{L}(\alpha, p, \infty, r) \leq\left(\frac{b_{\alpha}}{(2 r+2 \alpha+2)^{2-p}(4 r+2 \alpha+2)^{p-1}}\right)^{1 / p}, \quad 1 \leq p \leq 2$;

6) $\mathcal{L}(\alpha, p, q, r) \leq(\mathcal{L}(\alpha, p, p, r))^{p / q}(\mathcal{L}(\alpha, p, \infty, r))^{1-p / q}, \quad 0<p \leq q \leq \infty$.

Неравенство 5 доказывается аналогично случаю $r=0$ [7, предложение 1] из равенства

$$
D_{\alpha}^{r} f(0)=(-1)^{r} \int_{0}^{1} f(t) \mathcal{H}_{\alpha}\left(\chi_{r}\right)(t) d \nu_{\alpha}(t), \quad \chi_{r}(t)=t^{2 r} \chi_{[0,1]}(t) .
$$

Оно следует при $1 \leq p \leq 2$ из теоремы Пэли-Винера для преобразования Ганкеля и $(2.2)$. Теперь, как и в [7], можно применить неравенство Гёльдера и воспользоваться неравенствами

$$
\left\|\mathcal{H}_{\alpha}\left(\chi_{r}\right)\right\|_{p^{\prime}, d \nu_{\alpha}} \leq\left\|\mathcal{H}_{\alpha}\left(\chi_{r}\right)\right\|_{2, d \nu_{\alpha}}^{2 / p^{\prime}}\left\|\mathcal{H}_{\alpha}\left(\chi_{r}\right)\right\|_{\infty}^{1-2 / p^{\prime}}=\left\|\chi_{r}\right\|_{2, d \nu_{\alpha}}^{2 / p^{\prime}}\left\|\mathcal{H}_{\alpha}\left(\chi_{r}\right)\right\|_{\infty}^{1-2 / p^{\prime}}
$$


где $p^{\prime}=p /(p-1) \geq 2$. Каждый из сомножителей справа легко оценить, и это дает 5 .

Оценка 6 вытекает из неравенства $\|f\|_{q, d \nu_{\alpha}} \leq\|f\|_{p, d \nu_{\alpha}}^{p / q}\|f\|_{\infty}^{1-p / q}$ и применима для произвольных констант Никольского - Бернштейна.

Из 1, 3-6 и теоремы 1 при $1 \leq p \leq q=\infty$ сразу получаем аналогичные результаты в многомерном случае для константы $\mathcal{L}_{d, \kappa}(p, \infty, r)=\mathcal{L}\left(\alpha_{\kappa}, p, \infty, r\right)$.

Например, из 3 для $\kappa=0$ получаем оценку для $\mathcal{L}_{d, 0}(p, \infty, 0)$ и после перенормировки оценку (1.3). Отметим, что из взаимосвязи констант мы не можем получить случай $\kappa \neq 0$, $p<1$, и нужно действовать напрямую.

Равенство $\mathcal{L}_{d, \kappa}(2,2, r)=1$ легко доказать напрямую. Мы не стараемся перенести неравенство 2 , поскольку при $p \neq 2, \infty$ оно нуждается в уточнении даже в одномерном случае.

2. Приведем список некоторых интересных, на наш взгляд, задач для весовых неравенств Никольского - Бернштейна. Некоторые из них кажутся достаточно сложными.

1) Доказать порядковое неравенство Бернштейна для однородного полинома от градиента Данкля $\nabla_{\kappa}$. Здесь соответствующий мультипликатор Данкля в общем случае не будет четным радиальным полиномом, что делает задачу непростой.

2) Доказать неравенство Бернштейна с неточной константой для лапласиана Данкля $\Delta_{\kappa}$ при $p<1$.

3) Доказать единственность или описать все экстремальные функции в многомерных задачах при $1 \leq p \leq \infty$, а не только на подмножестве радиальных функций.

4) Вычислить точную весовую константу Бернштейна в каком-нибудь случае, отличном от $p=2, \infty$.

5) Вычислить многомерные константы Никольского - Бернштейна на подмножествах неотрицательных функций. В частных случаях это было сделано в [4] и отмеченной работе первого автора (Тр. Ин-та математики и механики УрО РАН. 2018).

\section{СПИСОК ЛИТЕРАТУРЫ}

1. Арестов В.В. О неравенствах С.Н. Бернштейна для алгебраических и тригонометрических полиномов // Докл. АН СССР. 1979. Т. 246, №6. С. 1289-1292.

2. Арестов В.В. О неравенстве разных метрик для тригонометрических полиномов // Мат. заметки. 1980. T. 27, № 4. C. 539-547.

3. Arestov V., Babenko A., Deikalova M., Horváth Á. Nikol'skii inequality between the uniform norm and integral norm with Bessel weight for entire functions of exponential type on the half-line // Anal. Math. 2018. Vol. 44, no. 1. P. 21-42. doi: 10.1007/s10476-018-0103-6.

4. Dai F., Gorbachev D., Tikhonov S. Nikolskii constants for polynomials on the unit sphere [eresource]. 21 p. URL: https://arxiv.org/pdf/1708.09837.pdf.

5. Ganzburg M. Sharp constants of approximation theory. I. Multivariate Bernstein-Nikolskii type inequalities [e-resource]. 19 p. URL: https://arxiv.org/pdf/1901.04400.pdf.

6. Ganzburg M.I., Tikhonov S.Yu. On sharp constants in Bernstein-Nikolskii inequalities // Constr. Approx. 2017. Vol. 45, no. 3. P. 449-466. doi: 10.1007/s00365-016-9363-1 .

7. Горбачев Д.В., Добровольский Н.Н. Константы Никольского в пространствах $L^{p}\left(\mathbb{R},|x|^{2 \alpha+1} d x\right) / /$ Чебышевский сб. 2018. Т. 19, № 2. С. 67-79. doi: 10.22405/2226-8383-2018-19-2-67-79.

8. Горбачев Д.В., Мартьянов И.А. О взаимосвязи констант Никольского для тригонометрических полиномов и целых функций экспоненциального типа // Чебышевский сб. 2018. Т. 19, № 2. С. 80-89. doi: 10.22405/2226-8383-2018-19-2-80-89

9. Gorbachev D.V., Ivanov V.I., Tikhonov S.Y. Positive $L^{p}$-bounded Dunkl-type generalized translation operator and its applications // Constr. Approx. 2018. P. 1-51.

doi: 10.1007/s00365-018-9435-5.

10. Gorbachev D.V., Ivanov V.I. Fractional smoothness in $L^{p}$ with Dunkl weight and its applications [e-resource]. 28 p. URL: https://arxiv.org/pdf/1812.04946.pdf. 
11. Иванов В.А. Точные результаты в задаче о неравенстве Бернштейна - Никольского на компактных симметрических римановых пространствах ранга 1 // Тр. МИАН СССР: сб. тр.: Исследования по теории дифференцируемых функций многих переменных и ее приложениям. Ч. 14. . Т. 194. М.: Наука, 1992. C. 111-119.

12. de Jeu M.F.E. Paley-Wiener theorems for the Dunkl transform // Trans. Amer. Math. Soc. 2006. Vol. 358. P. 4225-4250. doi: 10.1090/S0002-9947-06-03960-2.

13. Камзолов А.И. Об интерполяционной формуле Рисса и неравенстве Бернштейна для функций на однородных пространствах // Мат. заметки. 1974. Т. 15, № 6. С. 967-978.

14. Mejjaoli H., Trimèche K. On a mean value property associated with the Dunkl Laplacian operator and applications // Integral Transform. Spec. Funct. 2001. Vol. 12. P. 279-302. doi: 10.1080/10652460108819351 .

15. Levin E., Lubinsky D. Asymptotic behavior of Nikolskii constants for polynomials on the unit circle // Comput. Methods Funct. Theory. 2015. Vol. 15. P. 459-468. doi: 10.1007/s40315-015-0113-3.

16. Nessel R., Wilmes G. Nikolskii-type inequalities for trigonometric polynomials and entire functions of exponential type // J. Austral. Math. Soc. 1978. Vol. 25, no. 1. P. 7-18. doi: 10.1017/S1446788700038878.

17. Никольский С.M. Приближение функций многих переменных и теоремы вложения. Москва: Наука, 1977. 480 с.

18. Платонов С.С. Гармонический анализ Бесселя и приближение функций на полупрямой // Изв. РАН. Сер. математическая. 2007. Т. 71, № 5. С. 149-196.

19. Rösler M. A positive radial product formula for the Dunkl kernel // Trans. Amer. Math. Soc. 2003. Vol. 355. P. 2413-2438. doi: 10.1090/S0002-9947-03-03235-5.

Поступила 8.04.2019

После доработки 6.05.2019

Принята к публикации 13.05.2019

Горбачев Дмитрий Викторович

д-р физ.-мат. наук, профессор

Тульский государственный университет, г. Тула

e-mail:dvgmail@mail.ru

Иванов Валерий Иванович

д-р физ.-мат. наук, профессор

зав. кафедрой

Тульский государственный университет, г. Тула

e-mail: ivaleryi@mail.ru

\section{REFERENCES}

1. Arestov V.V. On inequalities of S.N. Bernstein for algebraic and trigonometric polynomials. Soviet Math. Dokl., 1979, vol. 20, no. 3, pp. 600-603.

2. Arestov V.V. Inequality of different metrics for trigonometric polynomials. Math. Notes, 1980, vol. 27, no. 4, pp. 265-269. doi: 10.1007/BF01140526.

3. Arestov V., Babenko A., Deikalova M., Horváth Á. Nikol'skii inequality between the uniform norm and integral norm with Bessel weight for entire functions of exponential type on the half-line. Anal. Math., 2018, vol. 44, no. 1, pp. 21-42. doi: 10.1007/s10476-018-0103-6 .

4. Dai F., Gorbachev D., Tikhonov S. Nikolskii constants for polynomials on the unit sphere. Available at: arXiv:1708.09837 [math.CA], 2017, 21 p.

5. Ganzburg M. Sharp constants of approximation theory. I. Multivariate Bernstein-Nikolskii type inequalities. arXiv:1901.04400 [math.CA], 2019, 19 p.

6. Ganzburg M.I., Tikhonov S.Yu. On sharp constants in Bernstein-Nikolskii inequalities. Constr. Approx., 2017, vol. 45, no. 3, pp. 449-466. doi: 10.1007/s00365-016-9363-1.

7. Gorbachev D.V., Dobrovolskii N.N. Nikolskii constants in $L^{p}\left(\mathbb{R},|x|^{2 \alpha+1} d x\right)$ spaces. Chebyshevskii Sbornik, 2018, vol. 19, no. 2, pp. 67-79 (in Russian). doi: 10.22405/2226-8383-2018-19-2-67-79 . 
8. Gorbachev D.V., Martyanov I.A. On interrelation of Nikolskii Constants for trigonometric polynomials and entire functions of exponential type. Chebyshevskii Sbornik, 2018, vol. 19, no. 2, pp. 80-89 (in Russian). doi: 10.22405/2226-8383-2018-19-2-80-89 .

9. Gorbachev D.V., Ivanov V.I., Tikhonov S.Y. Positive $L^{p}$-bounded Dunkl-type generalized translation operator and its applications. Constr. Approx., 2018, pp. 1-51. doi: 10.1007/s00365-018-9435-5.

10. Gorbachev D.V., Ivanov V.I. Fractional smoothness in $L^{p}$ with Dunkl weight and its applications. arXiv:1812.04946 [math.CA], 2019, 28 p.

11. Ivanov V.A. Precise results in the problem of the Bernstein - Nikol'skij inequality on compact symmetric Riemannian spaces of rank 1. Proc. Steklov Inst. Math., Investigations in the theory of differentiable functions of many variables and its applications, part 14, 1993, vol. 194, pp. 115-124.

12. de Jeu M.F.E. Paley-Wiener theorems for the Dunkl transform. Trans. Amer. Math. Soc., 2006, vol. 358, pp. 4225-4250. doi: 10.1090/S0002-9947-06-03960-2 .

13. Kamzolov A.I. On Riesz's interpolational formula and Bernshtein's inequality for functions on homogeneous spaces. Math. Notes, 1974, vol. 15, no. 6, pp. 576-582. doi: 10.1007/BF01152838.

14. Mejjaoli H., Trimèche K. On a mean value property associated with the Dunkl Laplacian operator and applications. Integral Transform. Spec. Funct., 2001, vol. 12, pp. $279-302$. doi: 10.1080/10652460108819351.

15. Levin E., Lubinsky D. Asymptotic behavior of Nikolskii constants for polynomials on the unit circle. Comput. Methods Funct. Theory, 2015, vol. 15, pp. 459-468. doi: 10.1007/s40315-015-0113-3.

16. Nessel R., Wilmes G. Nikolskii-type inequalities for trigonometric polynomials and entire functions of exponential type. J. Austral. Math. Soc., 1978, vol. 25, no. 1, pp. 7-18. doi: 10.1017/S1446788700038878.

17. Nikol'skii S.M. Approximation of functions of several variables and embedding theorems. Berlin; N Y: Springer-Verlag, 1975, 420 p. doi: 10.1007/978-3-642-65711-5 . Original Russian text (2nd ed.) published in Nikol'skii S.M. Priblizhenie funktsii mnogikh peremennykh i teoremy vlozheniya. Moscow: Nauka Publ., $1977,480 \mathrm{p}$.

18. Platonov S.S. Bessel harmonic analysis and approximation of functions on the half-line. Izvestiya: Math., 2007, vol. 71, no. 5, pp. 1001-1048. doi: 10.1070/IM2007v071n05ABEH002379.

19. Rösler M. A positive radial product formula for the Dunkl kernel. Trans. Amer. Math. Soc., 2003, vol. 355, pp. 2413-2438. doi: 10.1090/S0002-9947-03-03235-5.

Received April 8, 2019

Revised May 6, 2019

Accepted May 13, 2019

Funding Agency: This work was supported by the Russian Science Foundation (project no. 1811-00199).

Dmitry Viktorovich Gorbachev, Dr. Phys.-Math. Sci., Prof., Tula State University, Tula, 300012 Russia, e-mail: dvgmail@mail.ru .

Valerii Ivanovich Ivanov, Dr. Phys.-Math. Sci., Prof., Tula State University, Tula, 300012 Russia, e-mail: ivaleryi@mail.ru .

Cite this article as: D. V. Gorbachev, V. I. Ivanov. Nikol'skii-Bernstein constants for entire functions of exponential spherical type in weighted spaces. Trudy Instituta Matematiki $i$ Mekhaniki URO $R A N, 2019$, vol. 25, no. 2, pp. 75-87. 